\title{
HAND Gesture ReCognition USINg Statistical AND ARTificial GeOMETRIC Methods: A SuRVEY
}

\author{
Mokhtar M. Hasan \\ Computer Science Department, University of Baghdad, Iraq
}

\begin{abstract}
Gesture recognition represents the silent language that can be done with robots as well as they done to us, this overseas language ensures that everyone can understand the meaning of the gesturing as well as can reply and interact with. Because of that this silent language has chosen for deaf people in which can make their communication easier between each of them as well as with other people.

In this paper we have brought to the table two different outstanding gesture recognition systems, those two techniques achieved high ratio of recognition percentage as well as that are invariant-free techniques, especially rotation perturbation that hinders the achievement of high level recognition percentage, the first method is the recognition of hand gesture with the help of dynamic circle template and second one using variable length chromosome generic algorithm, these two methods has been applied to different people and the main objective was to reduce the database size used for training.
\end{abstract}

\section{KEYWORDS}

Gesture system, genetic algorithm, Gaussian Mixture Model, GMM, hand recognition.

\section{INTRODUCTION}

In the last three decades especially in 1970's, there were new born called computer and everyone was afraid from this new creature, now everyone almost can finish his day without spending several hours or at least one hour on this amazing device, however, the story of robots nowadays are much similar to the new computer at that time, but the difference is that the people started to attract any new technology, in order to make this robot familiar with the people a new language should be used, because of having hundreds of spoken languages each with sophisticated grammars, sign language was the only outlet to generalize the usage of the robots, face language can be used to communicate with robots but the problem is limited expression the face can offer, hand gesture seems the most potential element that can be used in this situation.

This hand gesture can be broken down into two categories:

- Firstly: non-geometric features in which all hand is interpreted regardless the useful detailed in the fluctuation of the fingers.

- Secondly: geometric features in which every single movement in the fingers the form the hand can yield a new meaning.

As seen; the geometric features can ensure the vast interpretations of meaningful gestures and in turns; can produce a long dictionary of gesture words. 
This sign language has an important usage when communication with hearing impaired people, as well as operating particular intelligent device [1], controlling over home appliances [1][2], playing video game and augmented reality as well [1], however, recently the robots are utilized for life support as human-friendly applications [3], furthermore, playing an intermediate interface between real and cyber world [3].Furthermore, gesture language represents the first language that the new-born children can speak in which they are unable to use their utterance to express their demands yet [4].

The difference between the posture and gesture is that, static gestures with no movement are called postures and generally requires less computation complexity [5] while the dynamic one is called the gestures which have some sort of movement and requires massive computation as compared with postures, it is obvious that the real time environment needs gestures more than postures [6]. The glove device are considered very simple form of gestures and have the advantage of location hand fingers simultaneously since each finger has its own identification such as glove devices [7][8], colored markers [6], or visual appearance image [6].

However, bared hand gesture are considered the most sophisticated in locating the fingers as well as hand palm and other hand parameters and most desirable since it is completely natural [9] and cost-free as compared with glove based techniques and without any cumbersome wires for connection as well as disease-free since using same glove for many objects can transfer different kinds of disease infections. Another reviews can be found in [10][11][12][13][14] and [15].Figure 1 shows the input devices for gesture system [15].

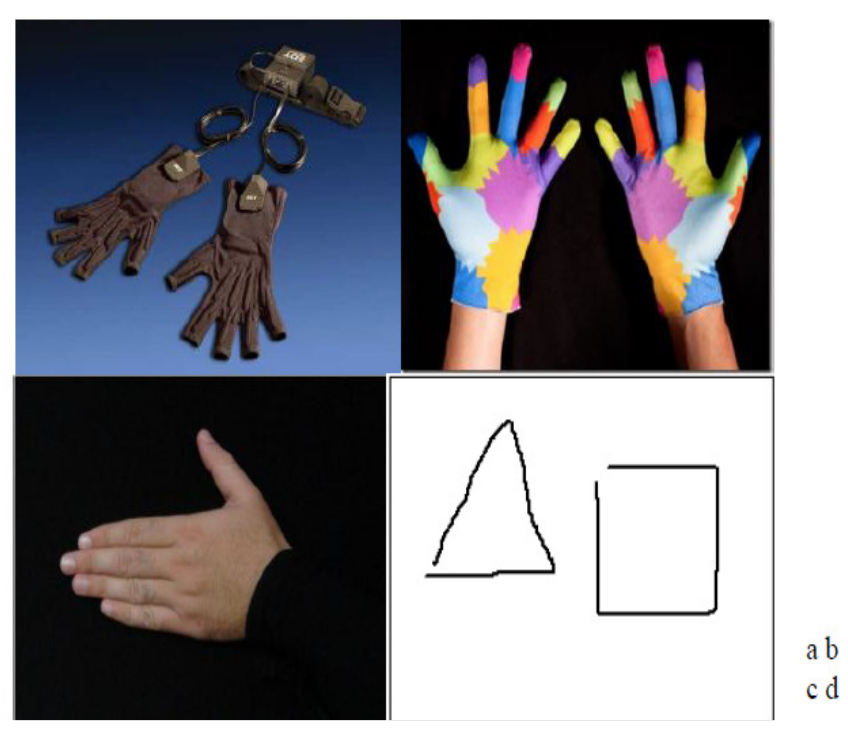

Figure 1: different devices for gesture system, a: data glove, b: marker, c: hand image, c: drawing.

We have presented herein two different papers, statistical based gesture recognition technique in [16] that based on dynamic circle template to split out the palm, fingers as well as wrist areas, and then features are extracted from these hand components in order to build a model using GMM. In the other hand, variable length chromosome is used in [17] for finding the palm, fingers and wrist area and after that GMM is used to locate fingers of the hand and decision tree is used for gesture understanding. 


\section{STATISTICAL BASED GESTURE RECOGNITION}

In this case, the feature extracting phase can be illustrated pictorially as follows:

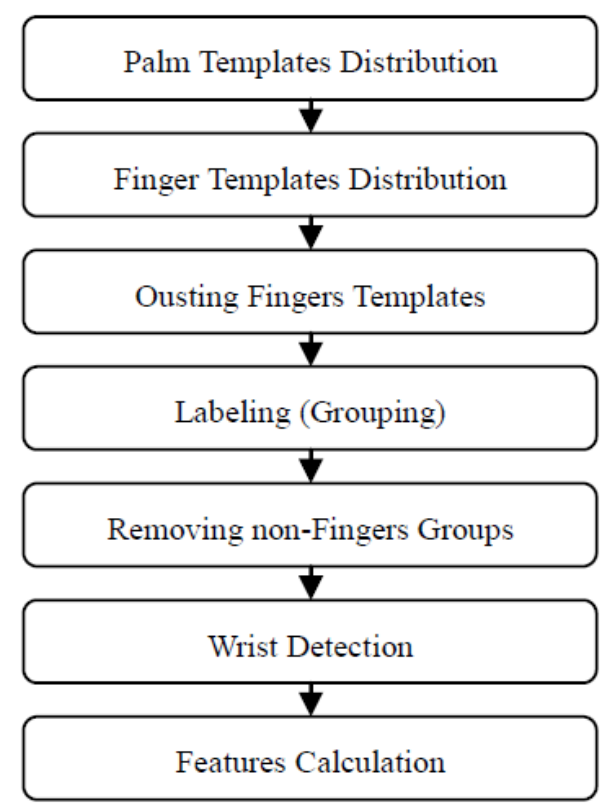

Figure 2: Feature extraction phase [16].

Consider the hand in Figure 3 (a), the main problem is how to locate the wrist area regardless of the direction applied, after finding the biggest circle that can fit in the human palm as figure 3 (b), the first angle and last angle of the wrist can be detected easily as figure 3 (c) by detecting the middle point of the peak area as figure 4 , this middle point is the wrist centre.

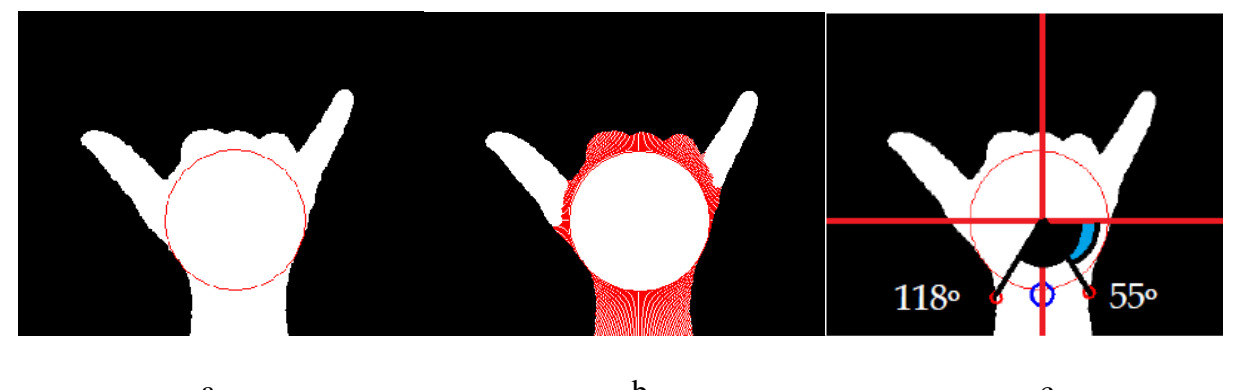

a

b

$\mathrm{C}$

Figure 3: determining the wrist centre [16], a: biggest circle can fit in the human palm, b: spotting the outside area of the circle, c: spotting out the wrist centre. 


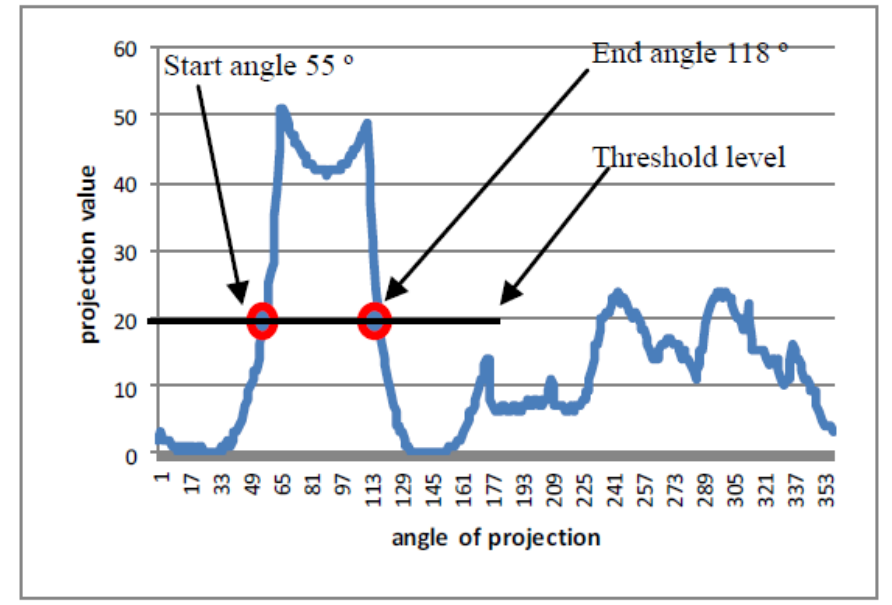

Figure 4: projection from the centre of the biggest circle to the boundaries of the human hand to find the wrist centre [16].

We have examined this method and get $100 \%$ spotting of the wrist centers with different rotated images; with and without sleeves as well.

\subsection{Features Applied}

Four features are applied in this paper, PD, BA, BsA and BB as listed in [16], these features are used subsequently in the classifier which is GMM, the following figure shows these features.
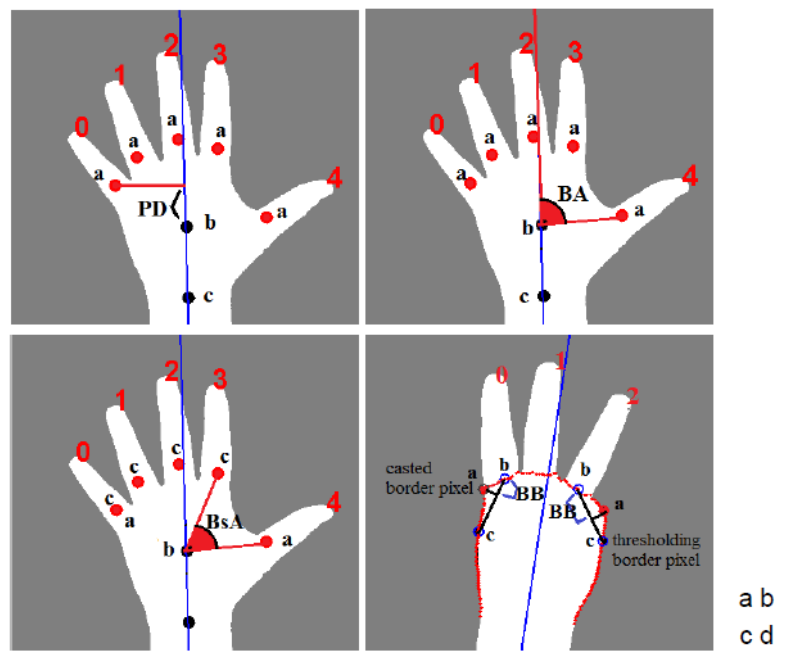

Figure 4: Features applied in [16] as labelled in the figure.

And the corresponding feature tables are listed in [16] in which the mean and variance represent the coefficient of Gaussian classifier, these features proved their immunity for any overlapping in the feature space. 


\subsection{Training and Testing}

A total of 26 different gestures are used for training purposes, this number is very low as compared to other systems since in this 26 different gestures a complete model has built for recognition different combination of fingers in the human hand, $(\mathrm{x}, \mathrm{n})$ refers to finger $\mathrm{x}$ trained with $\mathrm{n}$ samples, so, the training set is:

Training Set $=\{(1,10),(2,5),(3,5),(5,6)\}$

Similarly, testing set can be shows below:

Testing Set $=\{(0,2),(1,17),(2,25),(3,24),(4,24),(5,8)\}$

That gives total of 100 different gestures that used for testing; this system achieved is 407 milliseconds ( 0.407 second) per tested gesture with Gesture-wise $97.8 \%$ recognition percentage and Finger-wise $98.5 \%$ recognition percentage.

\section{VARIABLE LENGTH Chromosome for GeStuRE RECognition}

Genetic algorithm is applied in [17] in order to find the circle that the hand can fit, figure 5 shows this case.

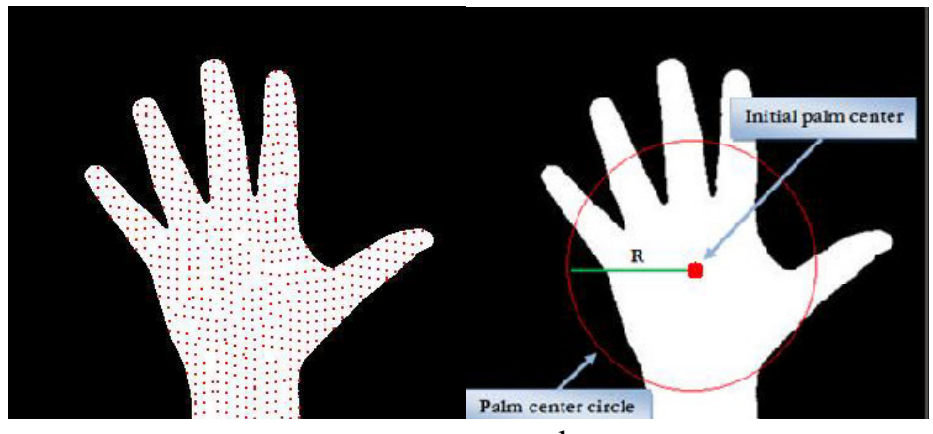

a

b

Figure 5: hand centre locating using variable length chromosome [17].

Wrist area are located using Euclidian distance between the start and end points of any segmented region, the biggest distance To locate the wrist area, Euclidean distance used to measure the distance difference between the start point and end point of the segmented regions and the larger difference represents the wrist area as seen in the following figure.
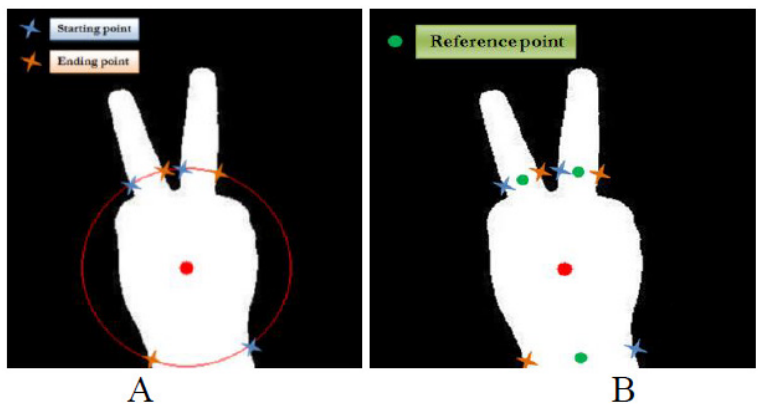

Figure 6: wrist area detection, a: locating start and end points, b: finding the centers. 


\subsection{Features Applied}

In this case, finger-base and fingertip are extracted firstly in order to prepare the model for the gesture system since good features mean good model which leads to good recognition performance, as following:

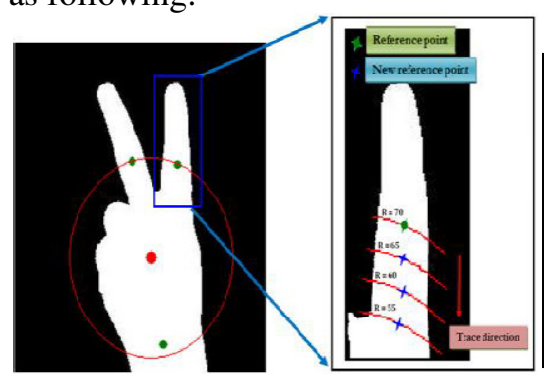

a: finger base detection.

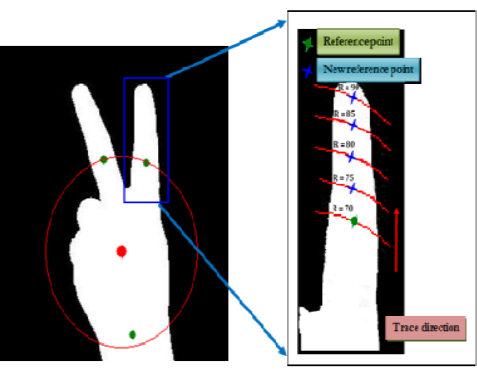

b: fingertip detection.

Figure 7: The operation of extracting the finger base and tip respectively.

After that PD and BA featured are extracted in order to classify the fingers using Gaussian classifier as in [18].

\subsection{Training and Testing Phases}

After the model for hand gesture has built using the extracted features, 26 presented gestures for training and 55 different gestures for testing as follows:

Training set $=\{(1,10),(2,5),(3,5),(5,6)\}$

Testing set $=\{(1,5),(2,12),(3,16),(4,13),(5,9)\}$

\section{EXPERIMENTAL RESULTS}

Both techniques have the following output when the input gesture is presented as follows:

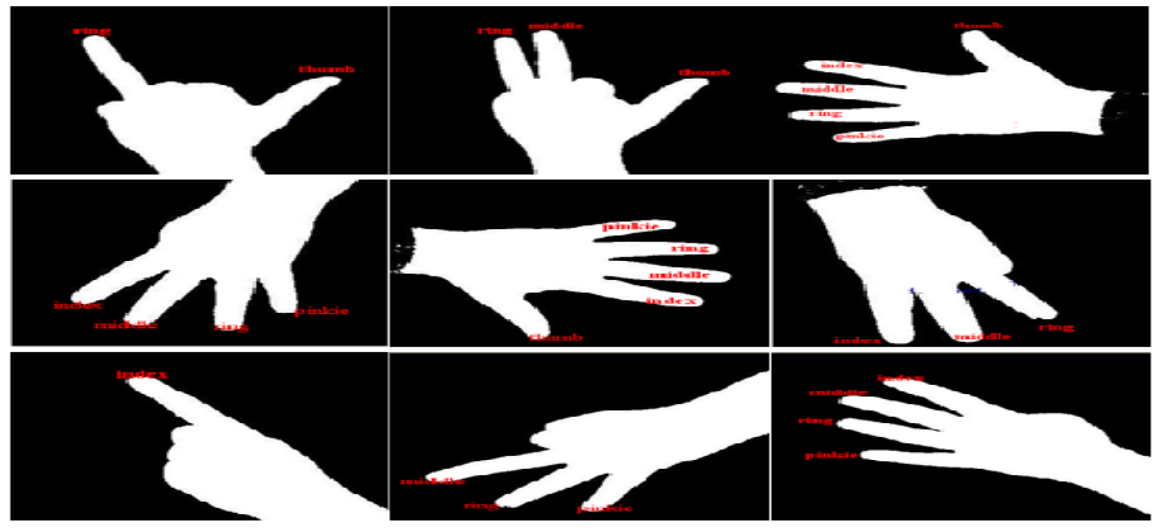

Figure 8: classification of fingers.

In which each finger is identified and labelled after the classification algorithm takes place automatically without any human-interference and regardless of the perturbations in the original input gesture as well as the rotation factor which has been managed completely. 


\section{CONCLUSiON}

Gesture system can act smoothly if we could reduce the database size using for training and these database should provide enough data as well, illumination challenge can be eliminated using normalized rgb, scaling and translation can be overcome by fixing the image size with trimming, rotation perturbation is the main obstacle that many researchers ignored it and they provides many training patterns for each single rotated object, this problem can be solved by unifying the object direction and produces features-rotated free for any given objects as seen in the previous demonstration and in Figure 8 as well. In this case the training database can be reduced dramatically and the speed of the training operation can be reduced as well so that a liable gesture recognition system can be generated.

\section{REFERENCES}

[1] Mokhtar M. Hasan, Pramod K. Mishra, "Real Time Fingers and Palm Locating using Dynamic Circle Templates", International Journal of Computer Applications, vol. 41 (6): 33-43, USA, March 2012 , doi: 10.5120/5547-7615

[2] RafiqulZaman Khan, Noor Adnan Ibraheem ,"Comparative Study and Analysis for Gesture Recognition Methodologies", International Journal of Advance Research in Science and Engineering, (e-ISSN 2319-8354), (p-ISSN 2319-8346), Volume 2(11), pp.64-69, India, November 2013.

[3] Noor A. Ibraheem, Rafiqul Z. Khan, "Novel Algorithm for Hand Gesture Modeling Using Genetic Algorithm with Variable Length Chromosome", International Journal on Recent and Innovation Trends in Computing and Communication, Vol. 2 (8):2175 - 2183, August 2014.

[4] Mokhtar M Hasan, "New Rotation Invariance Features Based on Circle Partitioning", Journal of Computer Engineering \& Information Technology, vol. 2 (2), USA, July 2013, doi: 10.4172/23249307.1000108.

[5] Xingyan Li. Gesture Recognition Based on Fuzzy C-Means Clustering Algorithm, Department of Computer Science. The University of Tennessee Knoxville, 2003

[6] S. Mitra,\& T. Acharya. Gesture Recognition: A Survey, IEEE Transactions on systems, Man and Cybernetics, Part C: Applications and reviews, Vol. 37, No. 3, pp 311- 324, doi: 10.1109/TSMCC.2007.893280, 2007.

[7] Pavlovic, V. I., Sharma, R. \& Huang, T. S., Visual Interpretation of Hand Gestures for HumanComputer Interaction: A Review. IEEE Transactions on Pattern Analysis And Machine Intelligence, Vol. 19, No. 7, pp 677- 695, doi; 10.1109/34.598226, 1997.

[8] Laura Dipietro, Angelo M. Sabatini \& Paolo Dario, Survey of Glove-Based Systems and their applications, IEEE Transactions on systems, Man and Cybernetics, Vol. 38, No. 4, pp 461-482, 2008.

[9] Simei G. Wysoski, Marcus V. Lamar, Susumu Kuroyanagi, \& Akira Iwata, A Rotation Invariant Approach On Static-Gesture Recognition Using Boundary Histograms And Neural Networks, IEEE 9th International Conference on Neural Information Processing, Singapura, 2002.

[10] RafiqulZaman Khan, Noor Adnan Ibraheem, Survey on Gesture Recognition for Hand Image Postures, International Journal of Computer and Information Science, Vol. 5, No. 3, pp 110-121. doi:10.5539/cis.v5n3p110, 2012.

[11] Joseph J. LaViola Jr., A Survey of Hand Posture and Gesture Recognition Techniques and Technology, Master Thesis, NSF Science and Technology Center for Computer Graphics and Scientific Visualization, USA, 1999.

[12] Thomas B. Moeslund, Erik Granum, A Survey of Computer Vision-Based Human Motion Capture, Elsevier, Computer Vision and Image Understanding, Vol. 81, pp 231-268, 2001.

[13] Ali Erol, George Bebis, MirceaNicolescu, Richard D. Boyle \&XanderTwombly, Vision-based hand pose estimation: A review, Elsevier Computer Vision and Image Understanding, Vol. 108, pp 52-73, 2007.

[14] RafiqulZaman Khan, Noor Adnan Ibraheem, "Geometric Feature Extraction For Hand gesture Recognition", International Journal of Computer Engineering \&Technology (IJCET), Volume 5 (7), pp.132-141, India, July 2014 


\section{Computer Applications: An International Journal (CAIJ), Vol.3, No.3, August 2016}

[15] Mokhtar M. Hasan, Pramod K. Mishra, "Hand Gesture Modeling and Recognition using Geometric Features: A Review", Canadian Journal on Image Processing and Computer Vision, vol. 3(1): 12-26, Canada, March 2012.

[16] Mokhtar M. Hasan, Pramod K. Mishra, "Novel Algorithm for Multi Hand Detection and Geometric Features Extraction and Recognition", International Journal of Scientific \& Engineering Research, vol. 3 (5): 1-12, France, May 2012.

[17] RafiqulZaman Khan, Noor Adnan Ibraheem,"Genetic Shape Fitting for Hand Gesture Modeling and Feature Extraction using Variable Length Chromosome", British Journal of Science, (ISSN 20473745), Volume 10 (1), UK, December 2013.

[18] Noor A. Ibraheem, "Finger Identification and Gesture Recognition Using Gaussian Classifier Model", International Journal of Applied Engineering Research, Scopus Indexed, vol.11 (10): 6924-6931, 2016.

[19] Mokhtar M. Hasan, Noor A. Ibraheem, "Automatic Block Selection for Synthesizing Texture Images using Genetic Algorithms”, Baghdad Science Journal, College of Science for Women, University of Baghdad, Iraq, vol. 6 (4): 822-830, December 2009. 